
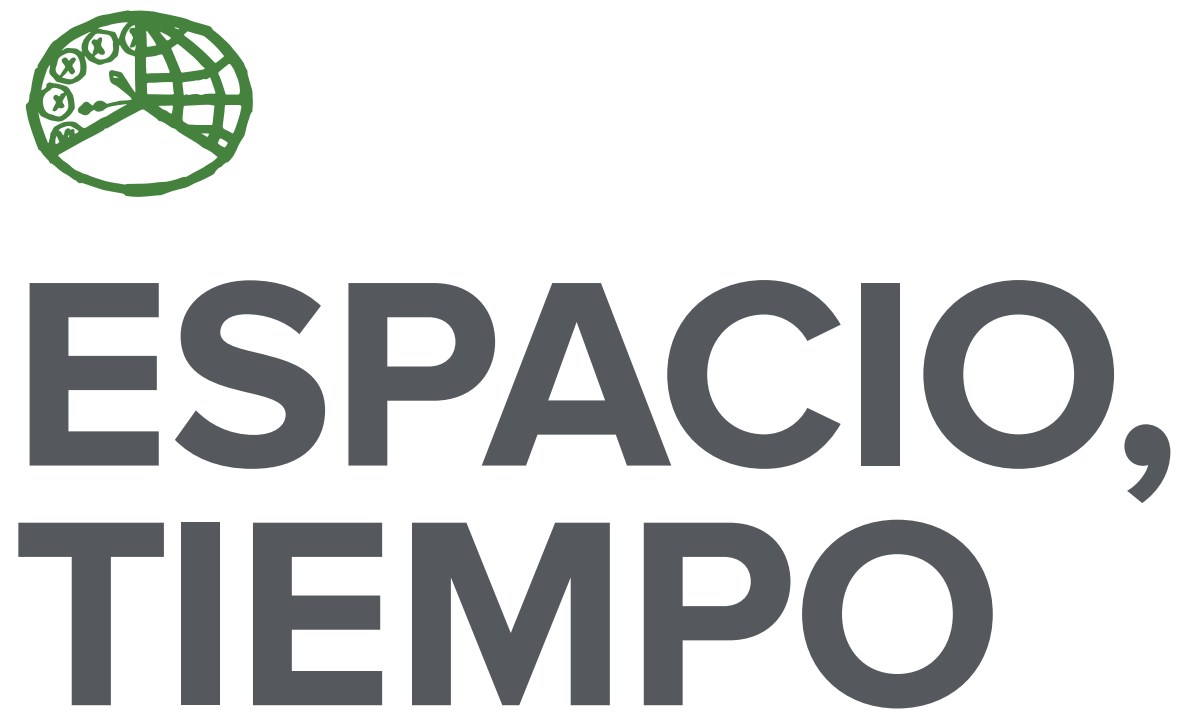

AÑOS 2015-2016

ISSN 1130-2968

E-ISSN 2340-146X
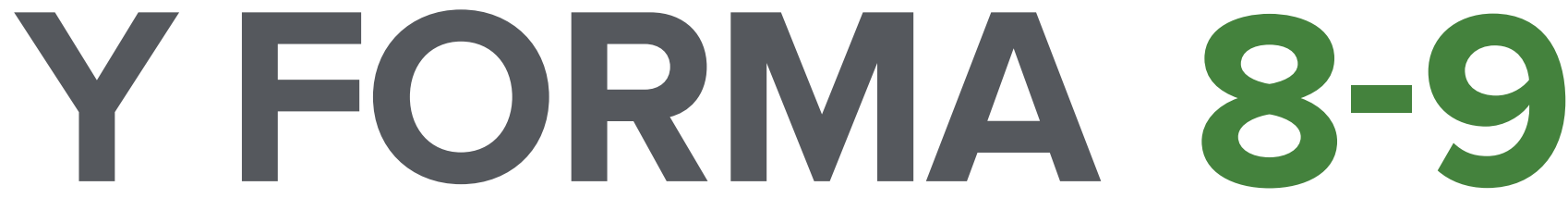

SERIE VI GEOGRAFÍA

REVISTA DE LA FACULTAD DE GEOGRAFÍA E HISTORIA 

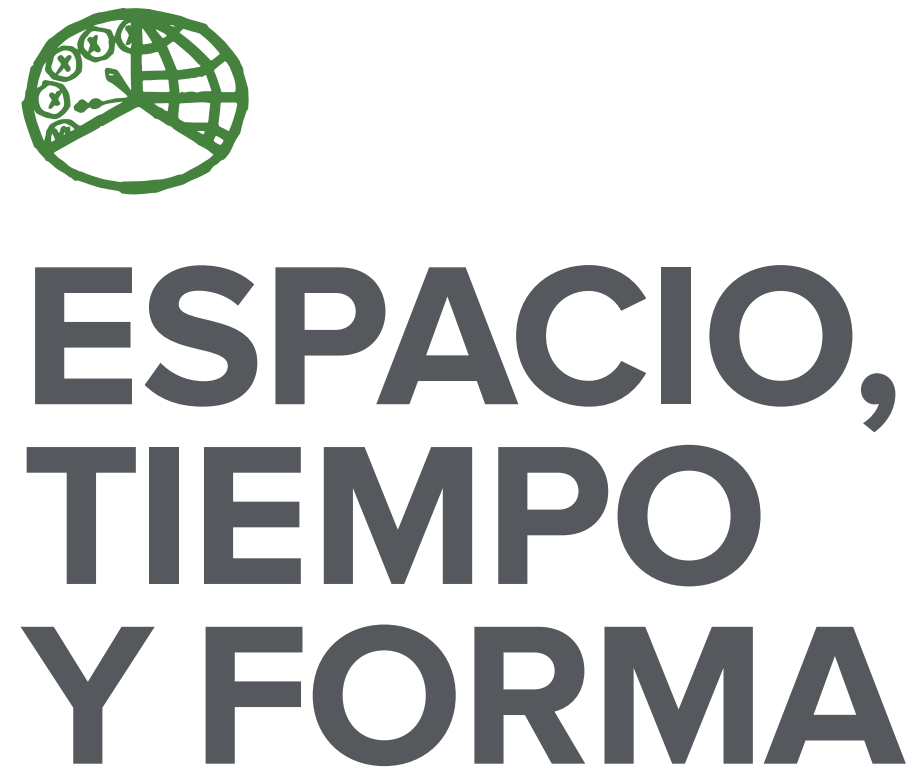

AÑOS 2015-2016

ISSN 1130-2968

E-ISSN 2340-146X

SERIE VI GEOGRAFÍA

REVISTA DE LA FACULTAD DE GEOGRAFÍA E HISTORIA

DOI: http://dx.doi.org/10.5944/etfvi.8-9.2016

\section{UกED}

UNIVERSIDAD NACIONAL DE EDUCACIÓN A DISTANCIA 
La revista Espacio, Tiempo y Forma (siglas recomendadas: ETF), de la Facultad de Geografía e Historia de la UNED, que inició su publicación el año 1988, está organizada de la siguiente forma:

$$
\begin{aligned}
& \text { SERIE I - Prehistoria y Arqueología } \\
& \text { SERIE II - Historia Antigua } \\
& \text { SERIE III - Historia Medieval } \\
& \text { SERIE IV - Historia Moderna } \\
& \text { SERIE V - Historia Contemporánea } \\
& \text { SERIE VI - Geografía } \\
& \text { SERIE VII - Historia del Arte }
\end{aligned}
$$

Excepcionalmente, algunos volúmenes del año 1988 atienden a la siguiente numeración:

$$
\begin{aligned}
& \mathrm{N}^{\circ} 1 \text { - Historia Contemporánea } \\
& \mathrm{N}^{\circ} 2 \text { - Historia del Arte } \\
& \text { N. }{ }^{\circ} 3 \text { - Geografía } \\
& \mathrm{N} .^{\circ} 4 \text { - Historia Moderna }
\end{aligned}
$$

ETF no se solidariza necesariamente con las opiniones expresadas por los autores.

Espacio, Tiempo y Forma, Serie vi está registrada e indexada, entre otros, por los siguientes Repertorios Bibliográficos y Bases de Datos: DICE, ISOC (CINDOC), RESH, IN-RECH, Dialnet, e-sPacio, UNED, CIRC, MIAR, FRANCIS, PIO, ULRICH'S, SUDOC, 2DB, ERIH (ESF).

\author{
UNIVERSIDAD NACIONAL DE EDUCACIÓN A DISTANCIA \\ Madrid, 2015-2016 \\ SERIE VI · GEOGRAFÍA N. ${ }^{\circ} 8-9,2015-2016$ \\ ISSN 1130-2968 · E-ISSN 2340-146x \\ DEPÓSITO LEGAL \\ M-21.037-1988 \\ URL \\ ETF VI · GEOGRAFÍA $\cdot$ http://revistas.uned.es/index.php/ETFVI \\ COMPOSICIÓN \\ Carmen Chincoa Gallardo · http://www.laurisilva.net/cch
}

Impreso en España · Printed in Spain

(c) (7) (8) Esta obra está bajo una licencia Creative Commons

Reconocimiento-NoComercial 4.0 Internacional. 


\title{
LAS SALINAS DE IMON (GUADALAJARA): UN PAISAJE CULTURAL DE GRAN VALOR HISTÓRICO
}

\author{
THE SALTWORKS OF IMON (GUADALAJARA): A \\ CULTURAL LANDSCAPE OF GREAT HISTORICAL \\ VALUE
}

Francisco José Morales Yago ${ }^{1}$

DOI: http://dx.doi.org/10.5944/etfvi.8-9.2016.16369

El paisaje del páramo castellano que une a través de la carretera comarcal CMIIo las localidades de Sigüenza y Atienza en la provincia de Guadalajara (Castilla-La Mancha), nos sorprende por tratarte de un espacio singular, único y de gran belleza: se trata de las más antiguas, grandes e importantes explotación de sal existente en la España interior, la denominada: Salinas de Imon. En su día este espacio fue el mayor centro de producción de sal del país y se configuró como uno de los centros neurálgicos del comercio de este mineral, creando un eje comercial y de riqueza que perduró hasta prácticamente su cierre en la década de los noventa del siglo XX, aunque lógicamente con la aparición del ferrocarril en el siglo XIX en nuestro país de forma más intensa este espacio perdió protagonismo productivo en relación a las salinas del litoral en donde los costes de producción de este mineral y su transporte resultó más competitivo.

Fueron concebidas como salinas de interior utilizando manantiales de agua salada que se obtienen al atravesar el agua los depósitos subterráneos de sal. Construidas en torno al siglo X, son las más antiguas de la Península. Desde su construcción los monarcas las utilizaron como medio de explotación para vender la sal obtenida a los nobles y el clero. Pero fue Alfonso VI quien finalmente concedió la explotación de las salinas al arzobispado de Sigüenza, quien se encargó de la extracción y el reparto y comercialización de la sal desde entonces. La importancia de estas salinas llevó a Carlos III, en el siglo XVIII, a ordenar su ampliación, construyendo nuevas norias, grandes almacenes, artesas y renovar el sistema de canales que aún se mantienen a pesar del actual estado de abandono.

La producción de la sal se realizaba durante todo el año, aunque los meses de mayor intensidad eran los comprendidos entre mayo y octubre, en esos meses la concentración de sal en las aguas subterráneas era mayor; ésta era extraída del subsuelo por medio de un sistema de pozos de cinco metros de profundidad que por medio de una noria octogonal permitía la extracción del agua que pasaba a los denominados recocederos, donde el agua era calentada antes de ser suministrada a las piscinas donde se depositaba la sal. El conjunto de las salinas lo formaban los

1. Universidad Nacional de Educación a Distancia.<fjmorales@geo.uned.es>. 
almacenes para el depósito de la sal, las piscinas y estanques donde se depositaba la sal; los recocederos lugar donde se calentaba el agua que se extraía del subsuelo, las norias y pozos que permitían la extracción, los canales por donde circulaba el agua hasta las piscinas y las regueras que servían como desagües del agua sobrante.

El conjunto de las piscinas está realizado en sillería y mampostería, tanto en los muros laterales como en su fondo. Disponen además de caminos empedrados con canto rodado que dan acceso a todas ellas y además establecen un espacio relativamente ancho donde acumular la sal que se sacaba de ellas. Las divisiones entre piscinas se realizan a través de tablazón que permite un fácil limpiado y posibilita la extracción de sal.
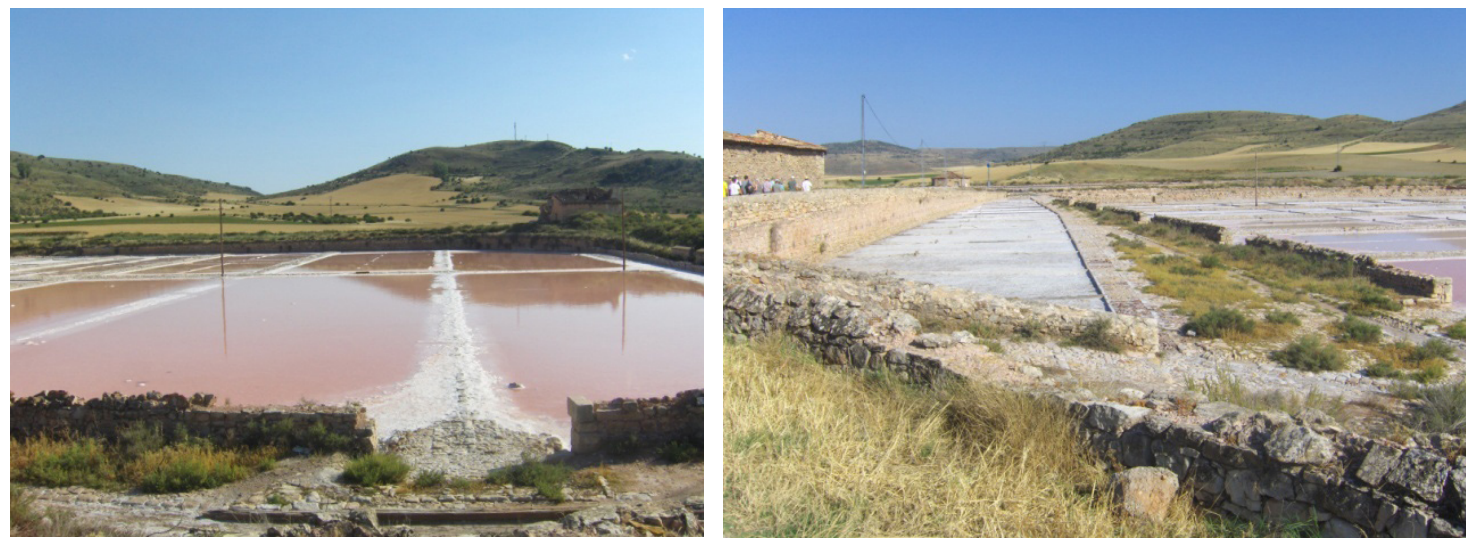

VISTAS DE LAS PISCINAS Y PARAMENTOS DE LAS SALINAS DE IMÓN.

En el siglo XX muchos de los canales construidos en madera fueron sustituidos por tuberías de cemento, aunque aún hoy se pueden ver algunos canales que mantienen su estructura en madera y permiten al visitante poder hacerse una idea fiel de cómo debieron ser sus canalizaciones de agua. Entre las piscinas se construyeron pequeñas acequias que facilitaban el desecado de las piscinas. Dentro de la edificación, las norias presentan una planta octogonal con una estructura de madera que se enlaza en el vértice de la cubierta, lo que permite un espacio completamente diáfano. De las cinco existentes, solo una conserva el cazo de barro, el sistema de engranajes de madera y el piso tratado para que diera vueltas el animal.

Al visitar este espacio nos invade una sensación de abandono y desolación puesto que los llamados almacenes de San Antonio y San José son los más antiguos de los que se conservan y están en un estado de ruina muy avanzado. Dispuestos de una base estructural a base de pórticos soportados por pies de madera y una entreplanta a base de suelo y viguería de madera que permite en paso a los vehículos que tenían que acceder para depositar la sal dentro del almacén. El almacén de San Antonio, de planta rectangular, conserva íntegro un pórtico de entrada, compuesto de grandes columnas de piedra ochavadas, y su rampa de acceso trasera a la entreplanta. Tiene adosada una chimenea de la pequeña central eléctrica que daba servicio a las salinas. El almacén de San José, de planta casi cuadrada, presenta dos edificaciones adosadas en su fachada principal y que conforma el acceso principal, realizadas a 
principios del siglo XX. En su acceso trasero conserva la torre con parte de la maquinaria que ayudaba a subir las vagonetas por la rampa.

Otra de las estructuras que aún permanece es la casa de los guardeses que cuidaban de la salina durante todo el año para evitar posibles robos o problemas que pudieran derivarse de todo el proceso de la salinización. Los materiales que se usaron para la construcción del conjunto arquitectónico fue de sillería y mampuesto en para los muros, de madera en los interiores y cubiertas y para los tejados se usó cerámica.
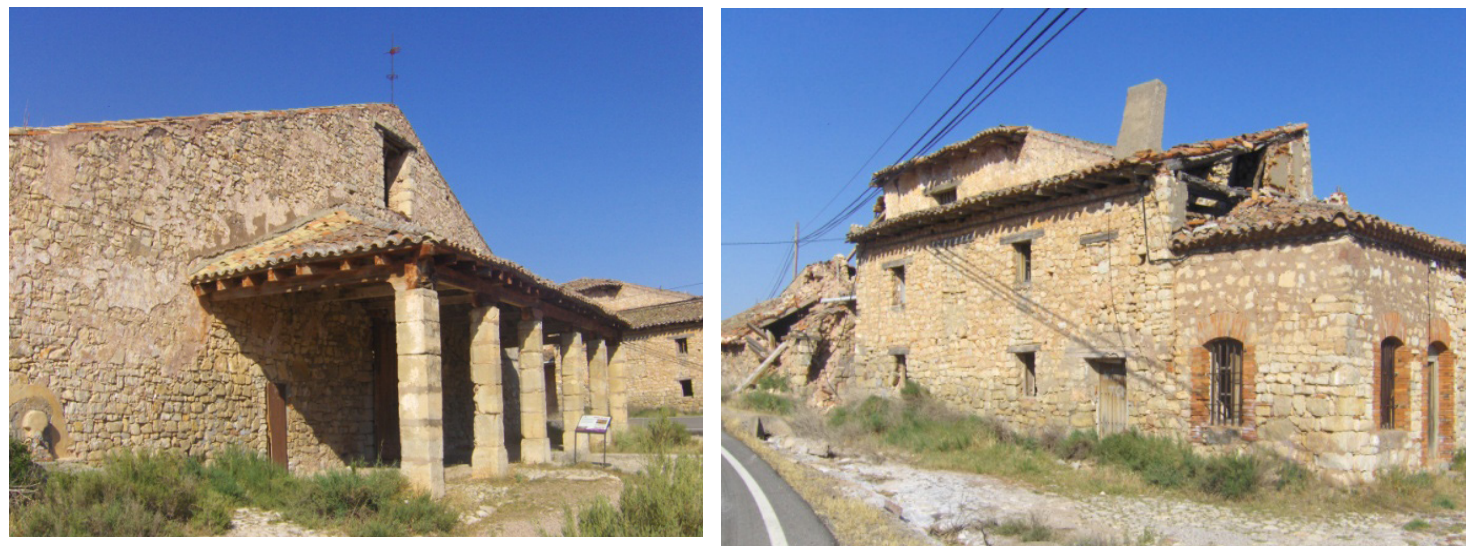

ESTADO DE RUINA DE LAS CONSTRUCCIONES DE LAS SALINAS DE IMÓN.

Actualmente las salinas de Imón están en desuso pero merece una visita para aprender cómo fue el funcionamiento de la extracción de sal en la que fue hasta casi la actualidad, la salina más importante de toda la Península Ibérica. Se trata de un espacio con un enorme valor cultural y turístico que debería recuperarse de forma integral. Sería preciso construir un centro de recepción de visitantes y un espacio para explicar todo el proceso de extracción de sal; solamente en las proximidades existe un hotel enclavado en un Caserón del siglo XVII que alberga habitaciones elegantes, restaurante, bar y spa con piscina exterior, también a 16 kilómetros se encuentra la ciudad de Sigüenza que alberga importantes recursos turísticos como la catedral y el castillo convertido actualmente tras su reconstrucción en parador nacional, en definitiva un conjunto de elementos que vale la pena conocer para los turistas asiduos a los espacios de interior en donde naturaleza, patrimonio y paisaje se unen en una perfecta simbiosis. 


\section{REFERENCIAS BIBLIOGRÁFICAS}

* <https://es.wikipedia.org/wiki/Salinas_de_Im\%C3\%B3n>

* <http://www.turismocastillalamancha.es/patrimonio/ salinas--siguenza--imon-85264/visita/>

* <http://www.saposyprincesas.com/actividad/guadalajara/aire-libre/rutas/ descubriendo-las-salinas-de-imon/> 


\section{SERIE VI GEOGRAFÍA}

REVISTA DE LA FACULTAD DE GEOGRAFÍA E HISTORIA
AÑOS 2015-2016

ISSN: $1130-2968$

E-ISSN 2340-146X

\section{8-9 ESPACIO, TIEMPO \\ Y FORMA}

14

PRESENTACIÓN · ForEWORD

\section{Artículos · Articles}

15

Carlos Alberto Abalerón

Diferencias innatas y desigualdades socio-espaciales de Calidad de Vida en San Carlos de Bariloche, Argentina / Innate differences and socio-spatial inequalities Quality of Life in San Carlos de Bariloche, Argentina

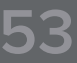

NATACHA CALVET TAPIA

Las prácticas lúdicas en la calle y la imagen del centro de la ciudad en Santiago de Chile / Recreational practices on the street and the image of the city center of Santiago de Chile

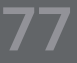

ENRIQUE dE Rosa GIOLITO

Las Complejas relaciones entre paisaje, puerto, ciudad y sus efectos en el patrimonio cultural en la bahía de Pasaia / The complex relationships between landscape, harbor, town and its effects on cultural heritage in the bay of Pasaia

\section{3} ANa María López ORTEgo

¿Quién está contra quién en los cerros orientales de Bogotá?. La perspectiva local desde el barrio la Cecilia / Who is against who in the eastern hills of Bogotá? - the local perspective from the neighborhood of la Cecilia

JULIO LÓPEZ-DAVALILLO LARREA

Las eurociudades rayanas / The ecocities bordering

\section{Beatriz Martínez de Teresa}

Estudio de los recursos hídricos en España: Análisis y caracterización en la Cuenca del Tajo / Study of water resources in Spain:Analysis and characterization in the Tajo Basin

Francisco José Morales YAgo

La incorporación de los portafolios electrónicos en el proceso enseñanza-aprendizaje y evaluación de la geografía en el alumnado de educación a distancia / The incorporation of electronic portfolios learning in geography and evaluation process in distance education students
24. MARcos ORTEga Montequín parado de tres casos en Asturias / Agrological potential in rural land planning; comparative study of three cases in Asturies

269 José Ramón SÁnchez Holgado

Las torres y atalayas de la costa tropical. ¿un recurso turístico pendiente de explotación? / The towers and watchtowers of the Costa Tropical.an Outstanding tourist resource exploitation

287

OSÉ RAMÓn SÁNCHEZ HOLGADO

Inundaciones en la rambla Albuñol. Causas y posibles medidas de intervención / Floods in Albuñol dry riverbed. Causes and possible intervention

\section{Reseñas · Book Review}

319 Yi-fu Tuan. (2015). Geografía romántica. En busca del paisaje sublime. Editado por Biblioteca nueva. Colección: Paisaje y teoría. Edición de Joan Nogué. 177 pp. ISBN: 978-84-16345-15-1 O (AURELIO NiETo CodinA)

323 Bosque Maurel, Joaquín (2011): Granada. Historia y cultura. Granada 323 Diputación Provincial de Granada-Libros de la Estrella, 210 pp. ISBN: 978-84-7807-510-2 (Alejandro García Ferrero)

\section{Imágenes y palabras · Pictures and words}

329 Francisco josé Morales Yago

Las salinas de Imón (Guadalajara): un paisaje cultural de gran valor histórico / The saltworks of Imón (Guadalajara): a cultural landscape of great historical value

\section{Historia de la Geografía Española · History of Geography in Spain}

335

Aurelio Nieto Codina, Dolores Brandis García, Carlos Pardo Abad Tres tesis doctorales dirigidas por don Manuel de Terán; el paisaje residencial en Madrid (Dolores Brandis), la enseñanza en Madrid (Ana Olivera) y el barrio de Salamanca (Rafael Más) / Three thesis directed by don Manuel Terán; residential landscape in Madrid (Dolores Brandis) teaching in Madrid (Ana Olivera) and the Salamanca district (Rafael Más) 